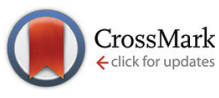

Cite this: Dalton Trans., 2015, 44, 13814

Received 29th June 2015, Accepted 7th July 2015

DOI: $10.1039 / \mathrm{c} 5 \mathrm{dt} 02458 \mathrm{~g}$

www.rsc.org/dalton

\section{Bismuth coordination networks containing deferiprone: synthesis, characterisation, stability and antibacterial activity $\uparrow$}

\author{
Andrew D. Burrows, ${ }^{* a}$ Monika Jurcic, ${ }^{a}$ Mary F. Mahon, ${ }^{\text {a }}$ Sandrine Pierrat, ${ }^{a}$ \\ Gavin W. Roffe, ${ }^{\mathrm{b}}$ Henry J. Windle ${ }^{\mathrm{c}}$ and John Spencer ${ }^{\star b}$
}

\begin{abstract}
A series of bismuth-dicarboxylate-deferiprone coordination networks have been prepared and structurally characterised. The new compounds have been demonstrated to release the iron overload drug deferiprone on treatment with PBS and have also been shown to have antibacterial activity against $H$. pylori.
\end{abstract}

Coordination network structures have attracted considerable attention in recent years. ${ }^{1,2}$ Although much of this interest has arisen from the capacity of this type of material to store gases within the pores, there has been an increasing interest in the inclusion of larger, more complex molecules including those with biological activity. Thus, metal-organic frameworks (MOFs) capable of storing drug molecules within pores and delivering these to an organism have been developed. ${ }^{3}$ An alternative strategy makes use of the active ingredient as a ligand bound to the metal within the framework. ${ }^{4-8}$ In this case, there is no requirement for the framework to be porous, and conceptually, a plasma-stable MOF could be fine-tuned for oral delivery, as a suspension, releasing the bioactive cargo under conditions (e.g. $\mathrm{pH}$ ) which digest the framework structure into its constituent parts.

Recently, we reported a series of zinc-based MOFs ${ }^{9}$ containing deferiprone, ${ }^{10}$ an iron overload drug, as an anionic chelating ligand. Immediate decomposition, and deferiprone release, was effected under mild conditions including in PBS (phosphate buffered saline) or dilute hydrochloric acid.

Bismuth compounds such as bismuth subsalicylate (BSS) and ranitidine bismuth citrate $(\mathrm{RBC})$ are generally poorly

\footnotetext{
${ }^{a}$ Department of Chemistry, University of Bath, Claverton Down, Bath BA2 7AY, UK. E-mail: a.d.burrows@bath.ac.uk; Tel: +44 (0)1225 386529

${ }^{b}$ Department of Chemistry, School of Life Sciences, University of Sussex, Falmer, Brighton, East Sussex BN1 9QJ, UK. E-mail: j.spencer@sussex.ac.uk; Tel: +44 (0)1273 877374

${ }^{c}$ Institute of Molecular Medicine, Trinity Centre for Health Sciences, Trinity College, Dublin 2, Ireland

$\dagger$ Electronic supplementary information (ESI) available: Syntheses, crystallographic studies, deferiprone release and antibacterial studies. CCDC 1051714-1051717. For ESI and crystallographic data in CIF or other electronic format see DOI: $10.1039 / \mathrm{c} 5 \mathrm{dt} 02458 \mathrm{~g}$
}

soluble and structurally ill-defined, but despite this, they are effective, orally-delivered drugs. ${ }^{11-15}$ When combined with antibiotics, (RBC also comprises a $\mathrm{H} 2$-antagonist, ranitidine), such drugs are extremely effective against Helicobacter pylori, which is implicated in peptic ulcers, gastritis and gastric cancers. ${ }^{16}$ Studies have elucidated the role of bismuth in inter alia disrupting enzymes such as urease (affecting nickel homeostasis and acid acclimation), mimicking iron deprivation and inhibiting cellular ATP synthesis. ${ }^{17}$ Antibacterial bismuth compounds are now finding biomedical uses such as in dental implants and haemodialysis catheters. ${ }^{18,19}$

The above uses and structural complexity of bismuth-based materials has prompted us to extend our studies to include bismuth complexes as model compounds for potential drug delivery and biomedical applications. It has been previously demonstrated that bismuth dicarboxylates can form coordination network structures. ${ }^{20-22}$ We therefore reasoned that it might be possible to include deferiprone into such structures, with the resultant compounds having a potential dual action mode. To this end we have investigated the formation and structures of bismuth-dicarboxylate-deferiprone complexes, then looked at their stability in terms of acting as deferiprone sources and also their activity against $H$. pylori.

The product from the reaction between bismuth(III) nitrate pentahydrate, 1,4-benzenedicarboxylic acid $\left(\mathrm{H}_{2} \mathrm{bdc}\right)$ and deferiprone (Hdfp) depends crucially on the $\mathrm{Bi}\left(\mathrm{NO}_{3}\right)_{3} \cdot 5 \mathrm{H}_{2} \mathrm{O}: \mathrm{Hdfp}$ ratio employed, with a mixture of DMF and water as solvent giving the best crystals. When the reaction is carried out with the $\mathrm{Bi}\left(\mathrm{NO}_{3}\right)_{3} \cdot 5 \mathrm{H}_{2} \mathrm{O}: \mathrm{Hdfp}$ ratio $1: 1$, the product consists of more than one phase, though pale yellow single crystals of $\left[\mathrm{Bi}_{2}(\mathrm{bdc})_{2}(\mathrm{dfp})_{2}\right] \cdot \mathrm{DMF} \mathbf{1}$ can readily be manually separated from grey/cream powders. When the reaction is carried out with the $\mathrm{Bi}\left(\mathrm{NO}_{3}\right)_{3} \cdot 5 \mathrm{H}_{2} \mathrm{O}: \mathrm{Hdfp}$ ratio $1: 4$, the reaction yields solely pale yellow crystals of $\left[\mathrm{Bi}_{2}(\mathrm{bdc})(\mathrm{dfp})_{4}\left(\mathrm{H}_{2} \mathrm{O}\right)_{2}\right] \cdot 2 \mathrm{DMF} 2$. Both 1 and 2 were characterised by X-ray crystallography, with powder X-ray diffraction confirming phase purity and ${ }^{1} \mathrm{H}$ NMR spectroscopy of the acid-digested samples confirming that the bdc: dfp ratio in the bulk is the same as that in the crystals. 
Reactions were also undertaken with the substituted dicarboxylic acids 2-amino-1,4-benzenedicarboxylic acid $\left(\mathrm{H}_{2}\right.$ bdc- $\left.\mathrm{NH}_{2}\right)$ and 2,5-dihydroxy-1,4-benzenedicarboxylic acid $\left(\mathrm{H}_{2}\right.$ bdc- $\left.(\mathrm{OH})_{2}\right)$. In both cases, the reactions with a $1: 1$ ratio of $\mathrm{Bi}\left(\mathrm{NO}_{3}\right)_{3} \cdot 5 \mathrm{H}_{2} \mathrm{O}$ : Hdfp gave mixed phases without single crystals analogous to those of 1 , but reactions with a $1: 4$ ratio of $\mathrm{Bi}\left(\mathrm{NO}_{3}\right)_{3} \cdot 5 \mathrm{H}_{2} \mathrm{O}: \mathrm{Hdfp}$ gave $\left[\mathrm{Bi}_{2}\left(\mathrm{bdc}-\mathrm{NH}_{2}\right)(\mathrm{dfp})_{4}\left(\mathrm{H}_{2} \mathrm{O}\right)_{2}\right] \cdot 2 \mathrm{DMF}$ 3 and $\left[\mathrm{Bi}_{2}\left\{\mathrm{bdc}-(\mathrm{OH})_{2}\right\}(\mathrm{dfp})_{4}\left(\mathrm{H}_{2} \mathrm{O}\right)_{2}\right] \cdot 2 \mathrm{DMF} \mathbf{4}$, both of which are isostructural to 2 .

The asymmetric unit of $\mathbf{1}$ consists of a bismuth centre, a dfp ligand and a bdc ligand, with residual electron density approximating to half of a molecule of DMF per bismuth centre. This gives rise to a formula for $\mathbf{1}$ of [Bi(bdc)(dfp)]-0.5DMF. The bismuth centre forms six $\mathrm{Bi}-\mathrm{O}$ contacts less than $2.65 \AA$, with coordination to a bidentate dfp ligand and two bidentate carboxylates. Three longer $\mathrm{Bi}-\mathrm{O}$ contacts, between $2.85 \AA$ and $2.95 \AA$, connect the bismuth centres into Bi-dfp-carboxylate chains (Fig. 1a). The dfp and carboxylate groups therefore adopt $\mu-\kappa^{1}, \kappa^{2}$ coordination modes, and overall the bismuth centre is 9-coordinate. The Bi-dfp-carboxylate chains are linked together by the benzene rings of the bdc ligands to form sheets (Fig. 1b). The dfp ligands project approximately perpendicular to these sheets, and those on neighbouring sheets interdigitate, though they are sufficiently offset to ensure there is no significant $\pi$-stacking. The dfp ligand atoms, oxygens excepted, are evenly disordered over two sites.

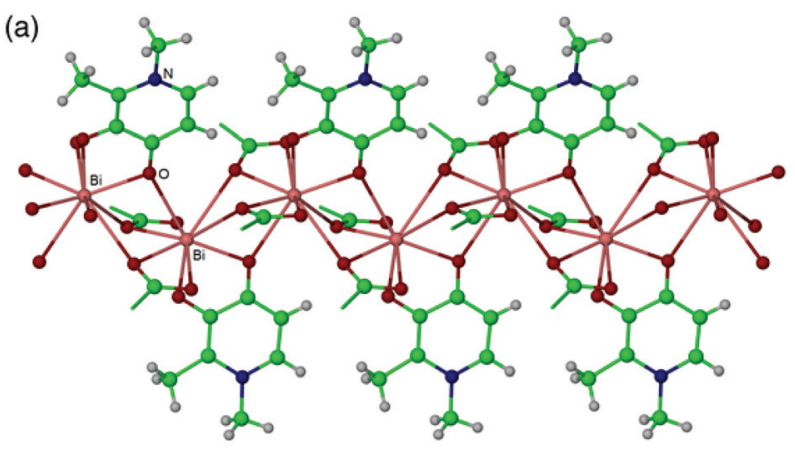

(b)

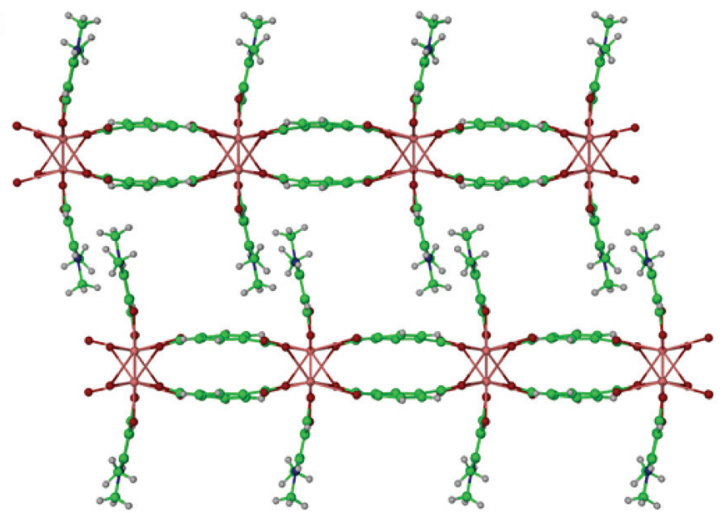

Fig. 1 The structure of [Bi(bdc)(dfp)]-0.5DMF 1, showing (a) the Bi-dfpcarboxylate chains, and (b) interdigitation of the sheets. Disorder omitted for clarity.
Compounds 2, 3 and $\mathbf{4}$ are isostructural, so only the structure of 2 will be described in detail. The asymmetric unit of 2 is composed of one bismuth centre, two dfp ligands, half of a bdc ligand, one included water molecule and one included DMF molecule. A centre of symmetry generates the remainder of the bdc ligand. The bismuth centre is coordinated to two bidentate dfp ligands and a bidentate carboxylate group, forming six $\mathrm{Bi}-\mathrm{O}$ bond distances of less than $2.75 \AA$. Additional longer $\mathrm{Bi}-\mathrm{O}$ contacts are present, with a bond to a carboxylate $\left[\mathrm{Bi}(1)-\mathrm{O}(2)^{\prime} 3.046(6) \AA\right]$ linking the bismuth centres into carboxylate-bridged dimers and a bond to a water molecule $[\mathrm{Bi}(1)-\mathrm{O}(7) 3.159(7) \AA]$ meaning that the bismuth centres are best described as eight-coordinate (Fig. 2a). Overall, the formula for 2 is $\left[\mathrm{Bi}_{2}(\mathrm{dfp})_{4}(\mathrm{bdc})\left(\mathrm{H}_{2} \mathrm{O}\right)_{2}\right] \cdot 2 \mathrm{DMF}$.

The bdc ligands bridge between the $\mathrm{Bi}_{2}(\mathrm{dfp})_{4}\left(\mathrm{O}_{2} \mathrm{CR}\right)_{2}\left(\mathrm{H}_{2} \mathrm{O}\right)_{2}$ units, linking these into chains running along the crystallographic $a$ axis, as shown in Fig. 2b. There is also offset $\pi$-stacking within the structure, with $\mathrm{dfp} \cdots$ bdc...dfp interactions present between the ligands within each chain $\left[\begin{array}{ll}3.3 & \AA\end{array}\right]$ and $\mathrm{dfp} \cdots \mathrm{dfp}$ interactions present between the ligands in neighbouring chains [3.2 ̊], serving to connect the chains into sheets. Hydrogen bonding is observed with the included water molecule acting as a hydrogen bond donor to both a dfp ligand and the included DMF molecule.

Previous studies on the use of zinc-based MOFs as a potential drug release platform for deferiprone showed immediate release in phosphate buffered saline (PBS) and HCl-PBS buffer ( $\mathrm{pH}$ 5.5) as monitored by LCMS. Compounds 2 and 4 were

(a)
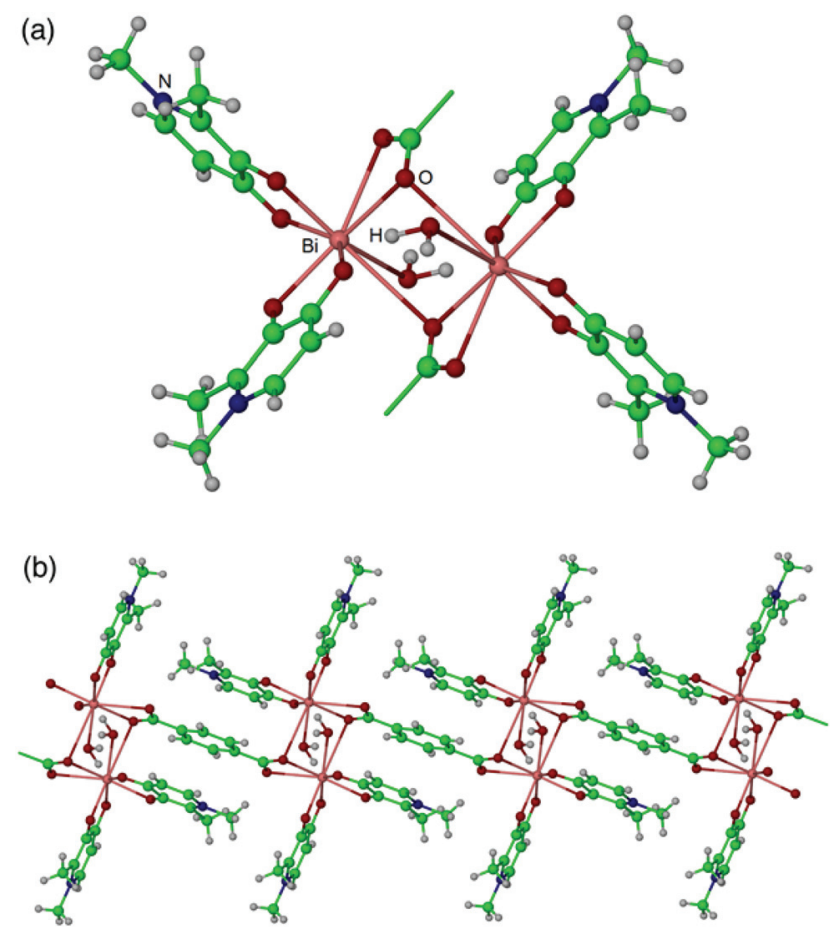

Fig. 2 The structure of $\left[\mathrm{Bi}_{2}(\mathrm{bdc})(\mathrm{dfp})_{4}\left(\mathrm{H}_{2} \mathrm{O}\right)_{2}\right] \cdot 2 \mathrm{DMF} 2$, showing (a) the $\left.\mathrm{Bi}_{2}(\mathrm{dfp})_{4}(\text { carboxylate })_{2} \mathrm{H}_{2} \mathrm{O}\right)_{2}$ dimers, and (b) the chains formed by linking together the dimers. 


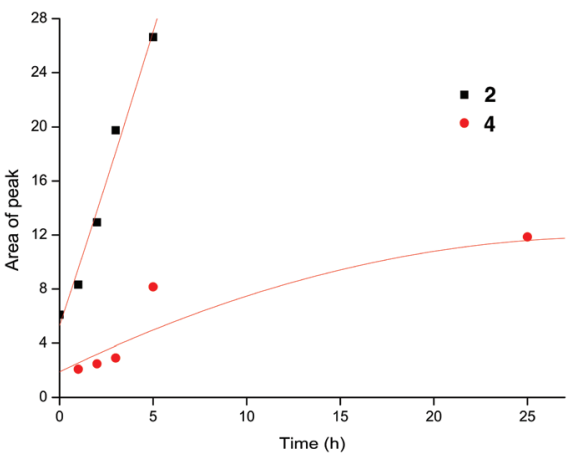

Fig. 3 LCMS stability study of $\left[\mathrm{Bi}_{2}(\mathrm{bdc})(\mathrm{dfp})_{4}\left(\mathrm{H}_{2} \mathrm{O}\right)_{2}\right] \cdot 2 \mathrm{DMF} 2$ and $\left[\mathrm{Bi}_{2}\left\{\mathrm{bdc}-(\mathrm{OH})_{2}\right\}(\mathrm{dfp})_{4}\left(\mathrm{H}_{2} \mathrm{O}\right)_{2}\right] \cdot 2 \mathrm{DMF} 4$ in PBS.

selected for analysis, and their stability studies were undertaken using ${ }^{1} \mathrm{H}$ NMR spectroscopy and LCMS.

$\left[\mathrm{Bi}_{2}(\mathrm{bdc})(\mathrm{dfp})_{4}\left(\mathrm{H}_{2} \mathrm{O}\right)_{2}\right] \cdot 2 \mathrm{DMF} 2$ showed fast release of deferiprone in $\mathrm{D}_{2} \mathrm{O}$ in NMR studies, which was mirrored in LCMS studies in PBS, over a period of $5 \mathrm{~h}$ (Fig. 3 , also see ESI $\dagger$ ). Interestingly, the deferiprone was released so rapidly in each respective medium that the ${ }^{1} \mathrm{H}$ NMR spectrum and LCMS trace taken immediately after addition already showed significant release. Identical studies were undertaken with $\left[\mathrm{Bi}_{2}\left\{\right.\right.$ bdc- $\left.(\mathrm{OH})_{2}\right\}-$ $\left.(\mathrm{dfp})_{4}\left(\mathrm{H}_{2} \mathrm{O}\right)_{2}\right] \cdot 2 \mathrm{DMF} \mathbf{4}$, and these showed no detectable release of deferiprone from $\mathrm{D}_{2} \mathrm{O}$ over a period of $24 \mathrm{~h}$, and significantly slower release than 2 in PBS as confirmed by LCMS (Fig. 3). To investigate the release of Hdfp from 4 using acid digestion, the LCMS trace was initially taken in PBS, showing little release, followed by $\mathrm{HCl}-\mathrm{PBS}(\mathrm{pH} 2)$ where substantial release was observed.

Compounds 2 and $\mathbf{4}$ were also tested against $H$. pylori (strain SS1) and compared with a positive control, bismuth subsalicylate (BSS). Different amounts of the compounds $(0,1$, 2, 4, 8, $1020 \mu \mathrm{g} \mathrm{ml}^{-1}$ ) were used to test their bacteriostatic and bactericidal activities. This was determined by spreading a lawn of an appropriately diluted suspension (post-exposure to the compounds for $24 \mathrm{~h}$ in brain heart infusion broth supplemented with foetal calf serum $(10 \%, \mathrm{v} / \mathrm{v})$ under microaerobic conditions) of the bacteria on Columbia blood agar plates followed by colony counting. Fig. 4 shows just the optical

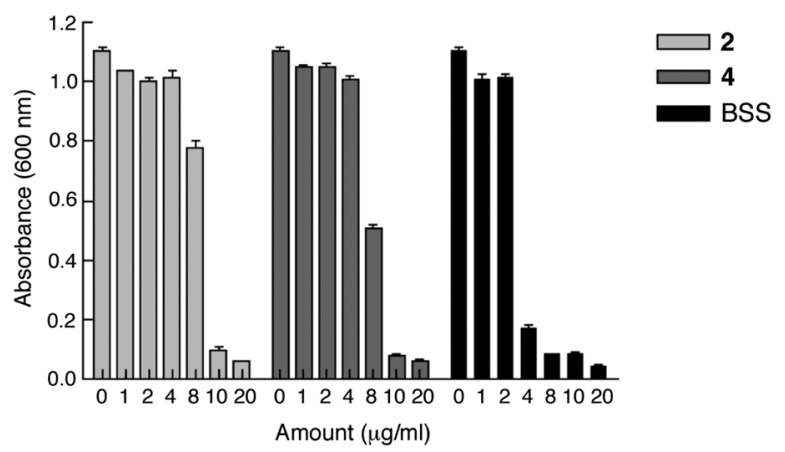

Fig. 4 Effects of 2 and 4 on $H$ pylori cultures in comparison to BSS. density readings $(600 \mathrm{~nm})$ of the bacterial suspensions $24 \mathrm{~h}$ post-treatment; as reduced readings relative to the control could represent either bactericidal or bacteriostatic activity the minimum inhibitory concentration (MIC) was determined. Both 2 and 4 showed MIC values of less than $10 \mu \mathrm{g} \mathrm{ml}{ }^{-1}$. In comparison, BSS showed a MIC value of less than $4.0 \mu \mathrm{g} \mathrm{ml}{ }^{-1}$. This indicates that $\mathbf{2}$ and $\mathbf{4}$ have appreciable H. pylori inhibitory action, comparable to that of BSS.

\section{Conclusions}

We have demonstrated that deferiprone can be incorporated as a terminal bidentate ligand into one- and two-dimensional bismuth coordination networks, and that it can be released on treatment with acid or PBS. Notably, the substituents on the 1,4-benzenedicarboxylate ligands play an important role in the rate of deferiprone release, with 2,5-dihydroxy substituents significantly retarding this. The reasons for this are currently under investigation. The coordination networks also show appreciable antibacterial activity, as demonstrated by inhibition of $H$. pylori growth. This potential dual mode of action, with both the ligand and metal centre playing different biological roles, has broad significance for other systems. Current work is focussing on removing the requirement for DMF in the syntheses and further retarding the rate of dissolution of the compounds in PBS as we move from the current model systems into compounds that are more suitable for in vivo studies.

The University of Bath, the University of Sussex, the R. M. Phillips Trust and SMSdrug.net are thanked for financial support, and Michael Paradowski is thanked for useful discussions.

\section{Notes and references}

1 C. Janiak and J. K. Vieth, New J. Chem., 2010, 34, 2366.

2 H. Furukawa, K. E. Cordova, M. O'Keeffe and O. M. Yaghi, Science, 2013, 341, 1230444.

3 P. Horcajada, R. Gref, T. Baati, P. K. Allan, G. Maurin, P. Couvreur, G. Férey, R. E. Morris and C. Serre, Chem. Rev., 2012, 112, 1232.

4 S. R. Miller, D. Heurtaux, T. Baati, P. Horcajada, J. M. Grenèche and C. Serre, Chem. Commun., 2010, 46, 4526.

5 B. Lou and F. He, New J. Chem., 2013, 37, 309.

6 J. An, S. J. Geib and N. L. Rosi, J. Am. Chem. Soc., 2010, 132, 38.

7 H. Su, F. Sun, J. Jia, H. He, A. Wang and G. Zhu, Chem. Commun., 2015, 51, 5774.

8 C. Tamames-Tabar, E. Imbuluzqueta, N. Guillou, C. Serre, S. R. Miller, E. Elkaïm, P. Horcajada and M. J. BlancoPrieto, CrystEngComm, 2015, 17, 456. 
9 A. D. Burrows, M. Jurcic, L. L. Keenan, R. A. Lane, M. F. Mahon, M. R. Warren, H. Nowell, M. Paradowski and J. Spencer, Chem. Commun., 2013, 49, 11260.

10 K. H. Thompson, C. A. Barta and C. Orvig, Chem. Soc. Rev., 2006, 35, 545.

11 R. Ge and H. Sun, Acc. Chem. Res., 2007, 40, 267.

12 H. Li and H. Sun, Curr. Opin. Chem. Biol., 2012, 16, 74.

13 T. E. Sox and C. A. Olson, Antimicrob. Agents Chemother., 1989, 33, 2075.

14 N. Yang and H. Sun, Coord. Chem. Rev., 2007, 251, 2354.

15 P. C. Andrews, G. B. Deacon, C. M. Forsyth, P. C. Junk, I. Kumar and M. Maguire, Angew. Chem., Int. Ed., 2006, 45, 5638.

16 R. Ge, Z. Chen and Q. Zhou, Metallomics, 2012, 4, 239.
17 M. V. Bland, S. Ismail, J. A. Heinemann and J. I. Keenan, Antimicrob. Agents Chemother., 2004, 48, 1983.

18 D.-J. Lin, M.-T. Tsai, T.-M. Shieh, H.-L. Huang, J.-T. Hsu, Y.-C. Ko and L.-J. Fuh, J. Biomater. Appl., 2013, 27, 553.

19 R. Schindler, U. Heemann, U. Haug, B. Stoelck, A. Karatas, C. Pohle, R. Deppisch, W. Beck and M. Hollenbeck, Nephrol., Dial., Transplant., 2010, 25, 2651.

20 A. C. Wibowo, M. D. Smith and H.-C. zur Loye, CrystEngComm, 2011, 13, 426.

21 A. Thirumurugan and A. K. Cheetham, Eur. J. Inorg. Chem., 2010, 3823.

22 A. Thirumurugan, J. C. Tan and A. K. Cheetham, Cryst. Growth Des., 2010, 10, 1736. 